\title{
Mice Lacking Central Serotonergic Neurons Show Enhanced Inflammatory Pain and an Impaired Analgesic Response to Antidepressant Drugs
}

\author{
Zhong-Qiu Zhao, ${ }^{1,2 *}$ Santina Chiechio, ${ }^{1,2 *}$ Yan-Gang Sun, ${ }^{1,2}$ Kai-Hua Zhang, ${ }^{1,2}$ Cheng-Shui Zhao, ${ }^{1,2}$ Michael Scott, ${ }^{6}$ \\ Randy L. Johnson, ${ }^{7}$ Evan S. Deneris, ${ }^{6}$ Kenneth J. Renner, ${ }^{8}$ Robert W. Gereau IV, ${ }^{1,2,5}$ and Zhou-Feng Chen ${ }^{1,2,3,4}$ \\ ${ }^{1}$ Washington University Pain Center and Departments of ${ }^{2}$ Anesthesiology, ${ }^{3}$ Psychiatry, ${ }^{4}$ Molecular Biology and Pharmacology, and ${ }^{5} \mathrm{Anatomy}$ and \\ Neurobiology, Washington University School of Medicine, St. Louis, Missouri 63110, ${ }^{6}$ Department of Neuroscience, School of Medicine, Case Western \\ Reserve University, Cleveland, Ohio 44106, ${ }^{7}$ Department of Biochemistry and Molecular Biology, M. D. Anderson Cancer Center, University of Texas Health \\ Science Center at Houston, Texas 77030, and ${ }^{8}$ Department of Biology, The University of South Dakota, Vermillion, South Dakota $57069^{6}$
}

\begin{abstract}
A large body of literature has implicated serotonin [5-hydroxytryptamine (5-HT)] in descending modulation of nociceptive transmission. Here, we have studied the pain behavior of $L m x 1 b$ conditional knock-out mice $\left(L m x 1 b^{f f / p}\right)$, which lack 5-HT neurons in the CNS. $L m x 1 b^{f f / p}$ mutant mice showed normal thermal and visceral pain responses but were less sensitive to mechanical stimuli and exhibited enhanced inflammatory pain compared with their littermate control mice. Importantly, the analgesic effect of several antidepressant drugs, including selective serotonin reuptake inhibitors (SSRIs), serotonin-norepinephrine reuptake inhibitors (SNRIs), and tricyclic antidepressants, was either abolished or greatly attenuated in $L m \times 1 b^{f f f / p}$ mice. Moreover, in the acute versus persistent pain settings, the analgesic actions of the SNRI duloxetine and the SSRI fluoxetine were differentially affected. Together, our results provide in vivo genetic evidence demonstrating that although the predominant role of the central 5-HT system in inflammatory pain is inhibitory, its role in acute mechanical pain is facilitatory. The findings that the analgesic effects of various antidepressant drugs are differentially dependent on the central 5-HT system should help us to understand the mechanism of the analgesic action of different classes of antidepressants in the management of persistent pain.
\end{abstract}

Key words: Lmx1b; serotonin; inflammatory pain; analgesic effect; antidepressant drugs; neurotransmitter

\section{Introduction}

Serotonin [5-hydroxytryptamine (5-HT)] in the CNS has long been implicated in the processing and modulation of pain and has been proposed to mediate both pronociceptive and antinociceptive actions at the spinal level via descending projections originating in the rostroventral medulla (Fields et al., 1991; Zhuo and Gebhart, 1991; Urban and Gebhart, 1999; Millan, 2002; Suzuki et al., 2004b). Numerous studies have been performed to identify the specific role of 5-HT in pain processing and modulation. These studies used a variety of approaches to either reduce serotonin levels in the CNS or block the site of action of this neurotransmitter, including the administration of 5-HT receptor agonists and antagonists, dietary tryptophan depletion, inhibition of

\footnotetext{
Received Jan. 4, 2007; revised May 2, 2007; accepted May 4, 2007.

This work was supported by National Institutes of Health (NIH) grants to R.W.G., E.S.D., R.L.J., K.J.R., and Z.-F.C. and by NIH Neuroscience Blueprint Core Grant P30 NS057105 to Washington University. We thank J. Yin, J. S. Wang, and C. Xiang for technical support, A. Basbaum for the $5-\mathrm{HT}_{10}$ antibody, and R. Blakely for the NET antibody. We also thank members of the Chen and Gereau laboratories, particularly Y. Carrasquillo, for helpful comments on this manuscript.

*Z.-Q.Z. and S.C. contributed equally to this work.

Correspondence should be addressed to Zhou-Feng Chen at the above address. E-mail: chenz@wustl.edu.

S. Chiechio's present address: Department of Pharmaceutical Sciences, University of Catania, 95125 Catania, Italy.

DOI:10.1523/JNEUROSCI.1623-07.2007

Copyright $\odot 2007$ Society for Neuroscience $\quad$ 0270-6474/07/276045-09\$15.00/0
}

serotonin biosynthesis, and selective lesions of serotonergic projections (Le Bars, 1988; Hamon et al., 1991). Despite the important findings obtained from these studies, possible nonspecific effects of pharmacological manipulations, the multiple sites of 5-HT action, the incomplete 5-HT depletion or lesions, and the adaptive changes in monoamine turnover have complicated the efforts to reach a consensus regarding the role of 5-HT even in a defined pain model (Hamon et al., 1991; Millan, 2002). Nevertheless, together with norepinephrine (NE), 5-HT has been recognized as one of the main neurotransmitters participating in endogenous supraspinal pain-modulating systems (Fields and Basbaum, 1978; Basbaum and Fields, 1984; Millan, 2002).

Consistent with this hypothesis, pharmacological manipulations that increase 5-HT and NE neurotransmission using tricyclic antidepressants (TCAs) such as amitriptyline and desipramine, which inhibit 5-HT and/or NE reuptake, are commonly used in the clinical management of chronic pain (Sindrup and Jensen, 1999; Collins et al., 2000; Lynch, 2001). The analgesic effect induced by antidepressant drugs has been postulated to occur primarily through inhibition of NE rather than 5-HT reuptake, with the latter augmenting the former (Fishbain et al., 2000). This hypothesis mainly arises from clinical trials indicating that efficacy of selective serotonin reuptake inhibitors (SSRIs), which affect only 5-HT levels, is lower than that of TCAs or 
is similar to placebo, whereas those affecting both 5-HT and NE have a better efficacy in relieving the pain symptoms (Max et al., 1992; Briley, 2004; Maizels and McCarberg, 2005). Indeed, more balanced NE/5-HT reuptake inhibitors (SNRIs), such as duloxetine, are showing promising results in treating the patients with diabetic peripheral neuropathic pain and fibromyalgia, supporting the important role of both 5-HT and NE in clinical pain management (Briley, 2004; Maizels and McCarberg, 2005; Wernicke et al., 2006). Despite these studies, the mechanism of action of the analgesic effect of these drugs is not well understood, especially with respect to persistent pain.

Lm $x 1 b$ is a LIM homeodomain-containing transcription factor and is the only transcription factor thus far identified as being absolutely required for the differentiation of all postmitotic 5-HT neurons in the CNS (Ding et al., 2003). We have previously generated a line of mice ( $L m \times 1 b^{f / f / p}$ mice) lacking the central 5-HT system but exhibiting normal locomotor activity (Zhao et al., 2006), which provide a new revenue for studying the function of 5-HT neurons in animal behaviors. The goal of the present study was to evaluate acute and inflammatory pain behaviors of $L m \times 1 b^{f / f / p}$ mice and to investigate the contribution of this neurotransmitter to the analgesic effect of several antidepressant drugs by using $L m \times 1 b^{f / f / p}$ mice.

\section{Materials and Methods \\ Animals}

$L m \times 1 b^{f f f / p}$ mice were genotyped as described previously (Zhao et al., 2006). Because our pilot studies indicated no significant molecular, anatomic, or behavioral differences between wild-type mice littermates and $L m \times 1 b^{f /+/ p}$ or $L m \times 1 b^{f /+}$ mice, wild-type or $L m x 1 b^{f /+/ p}$ or $L m x 1 b^{f /+}$ littermate mice were used as the control group in the present study, and the control mice were referred to as wild-type mice throughout the text. Wild-type and $L m x 1 b^{f / f / p}$ male mice aged between 8 and 12 weeks were acclimated to the experimental room and were used for behavioral tests by observers blind to both the genotype and the treatment of the animals. All experiments were done in accordance with experimental protocols approved by the Animal Studies Committee at Washington University School of Medicine.

\section{Behavioral experiments}

Thermal sensitivity. For the tail-flick test, a noxious heat stimulus was applied via a focused, radiant heat light source (IITC, Woodland Hills, CA) to the dorsal surface of the tail. The baseline tail-flick latency was calculated as the mean of three different measurements taken at $10 \mathrm{~min}$ intervals. For the Hargreaves test, thermal sensitivity was measured using a Hargreaves-type apparatus (IITC) (Hargreaves et al., 1988). Baseline of the paw-withdrawal latency was calculated as the mean of three to five different measurements taken at 15 min intervals.

Mechanical sensitivity. Mechanical sensitivity was assessed using a set of calibrated von Frey filaments (Touch-Test kit; Stoelting, Chicago, IL). Each filament was applied five consecutive times, and the smallest filament that evoked reflexive withdrawal of the paw on at least three of the five trials was taken as the paw-withdrawal threshold.

Capsaicin test. Capsaicin (Sigma-Aldrich, St. Louis, MO) $[1 \mu \mathrm{g} / 10 \mu \mathrm{l}$ of vehicle (10\% ethanol and $10 \%$ Tween 80 in saline)] or $10 \mu$ l of vehicle alone was injected into the plantar surface of one hindpaw, and the duration of licking and flinching of the injected paw was recorded for the first $5 \mathrm{~min}$. Paw-withdrawal threshold was tested with von Frey filaments before capsaicin injection (baseline) and at 30, 60, 120, and $180 \mathrm{~min}$ after the capsaicin injection.

Formalin test. Formalin $(5 \% ; 10 \mu \mathrm{l}$ in saline; Sigma-Aldrich) was injected into the plantar surface of one hindpaw, and the total time spent licking or flinching of the injected paw was monitored for $60 \mathrm{~min}$ and recorded in 5 min intervals.

Paw edema. A dial caliper was used to measure the change of the paw diameter before and $60 \mathrm{~min}$ after the capsaicin or formalin injection.

Carrageenan test. Carrageenan $(2 \% ; 20 \mu \mathrm{l}$ in saline; Sigma-Aldrich) was injected subcutaneously into the plantar surface of one hindpaw. Mechanical thresholds were measured before and $3 \mathrm{~h}$ after carrageenan injection, using von Frey filaments as described above.

Visceral pain. Acetic acid solution ( $0.6 \%$ in saline; Sigma-Aldrich) was slowly injected intraperitoneally at $5 \mathrm{ml} / \mathrm{kg}$ of body weight, and the number of writhes per animal was measured for $20 \mathrm{~min}$ after injection (Cao et al., 1998).

\section{Rescue experiments}

The effect of acute injection of 5-HT on capsaicin-induced inflammatory pain was evaluated in wild-type and $L m \times 1 b^{f / f / p}$ mice. Five microliters of various doses of 5-HT (in saline; Sigma-Aldrich) or saline alone were injected intrathecally 10 min before capsaicin injection, and the spontaneous response was observed as described above.

\section{Antidepressant drugs}

The doses of antidepressants were chosen according to previous studies (Singh et al., 2001; Iyengar et al., 2004; Jones et al., 2005) and our pilot studies, in which the drug dose that produced the maximum analgesic effect without causing toxicity or motor impairment was identified. Thermal and mechanical sensitivity and formalin-induced spontaneous nociceptive behavior were measured as mentioned above. To evaluate the effects of antidepressants on acute pain, thermal withdrawal latencies of wild-type and $L m \times 1 b^{f / f / p}$ mice after receiving an intraperitoneal administration of fluoxetine ( $20 \mathrm{mg} / \mathrm{kg}$; Tocris, Ellisville, MO), amitriptyline $(20 \mathrm{mg} / \mathrm{kg}$; Sigma-Aldrich) or duloxetine $(30 \mathrm{mg} / \mathrm{kg}$; Toronto Research Chemicals, North York, Ontario, Canada) were measured (Hargreaves test) every $30 \mathrm{~min}$ for a period of $3 \mathrm{~h}$. Results were expressed as percentage of maximum possible effect [\%MPE = (postdrug latency - predrug latency) $\times 100 /($ cutoff time - predrug latency) $]$. To examine the effects of antidepressants on persistent pain, mice were intraperitoneally injected with vehicle (saline), fluoxetine $(20 \mathrm{mg} / \mathrm{kg})$, or duloxetine $(30 \mathrm{mg} / \mathrm{kg}) 30 \mathrm{~min}$ before intraplantar injection of formalin or $90 \mathrm{~min}$ after intraplantar injection of carrageenan, and their spontaneous or mechanical pain behavior was measured.

\section{Immunocytochemical staining and in situ hybridization}

Immunocytochemical staining and in situ hybridization were performed as described previously (Ding et al., 2003). For immunocytochemistry, the sections were sequentially incubated with rabbit anti-5-HT $\mathrm{T}_{1 \mathrm{D}}$ receptor $(1: 10,000)$ in PBS containing $2 \%$ normal donkey serum and $0.3 \%$ Triton X-100 overnight, biotinylated donkey anti-rabbit (1:200; Jackson ImmunoResearch, West Grove, PA) for $2 \mathrm{~h}$, and Cy3-conjugated streptavidin (1:1000; Jackson ImmunoResearch) for $1 \mathrm{~h}$. After washing with PBS, the slides were observed under fluorescence microscopy.

\section{Semiquantitative reverse transcription- $P C R$}

Two micrograms of total RNA of each group were reverse transcribed by SuperScriptII Reverse Transcriptase (Invitrogen, Carlsbad, CA) with oligo $\mathrm{d}(\mathrm{T})_{12-18}$, and $1 \mu \mathrm{l}$ of cDNAs was used for semiquantitative reverse transcription (RT)-PCR. Glyceraldehyde-3-phosphate dehydrogenase $($ GAPDH $)$ was used as an internal control. The ratio of signal intensity of $5-\mathrm{HT}_{3 \mathrm{~A}}$ and GAPDH products was quantified using NIH ImageJ, and the data are presented as the mean of the ratio $(n=3)$. The paired primers for corresponding genes were as follows: $5-\mathrm{HT}_{3 \mathrm{~A}}(788 \mathrm{bp}), 5^{\prime}$ tgtatgccatcctcaacg- $3^{\prime}$ and $5^{\prime}$-actcagcgaggcttatcac- $3^{\prime}$; GAPDH (440 bp), $5^{\prime}$-aacgggaagcccatcacc- $3^{\prime}$ and $5^{\prime}$-cagccttggcagcaccag- $3^{\prime}$.

\section{Western blot}

The lumbar segment of the spinal cord of $L m \times 1 b^{f / f / p}$ and wild-type mice was removed and homogenized. Ten micrograms of total protein were separated by $10 \%$ SDS-PAGE and electrophoretically transferred onto protein-sensitive nitrocellulose membranes (Criterion blotter; Bio-Rad, Hercules, CA). The membrane was blocked in Odyssey blocker (LI-COR, Lincoln, NE) for $1 \mathrm{~h}$ and rabbit anti-norepinephrine transporter (NET) and mouse anti-MAP2 (microtubule-associated protein 2) (SigmaAldrich) primary antibody were simultaneously used for immunoblotting overnight at $4^{\circ} \mathrm{C}$. A goat anti-rabbit antibody labeled with IRD800CW and a goat anti-mouse antibody labeled with Alexa 680 (LICOR) were incubated for $1 \mathrm{~h}$ at room temperature. Proteins were de- 
A

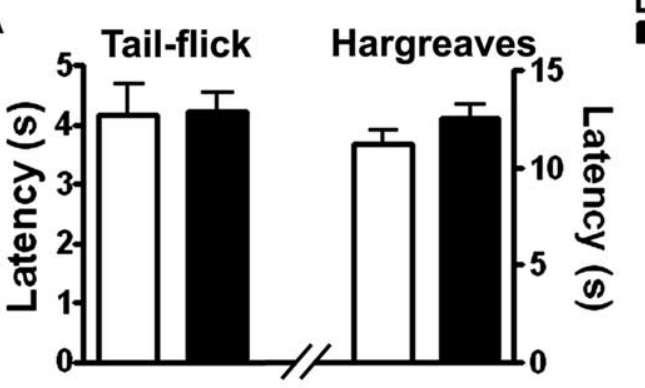

B

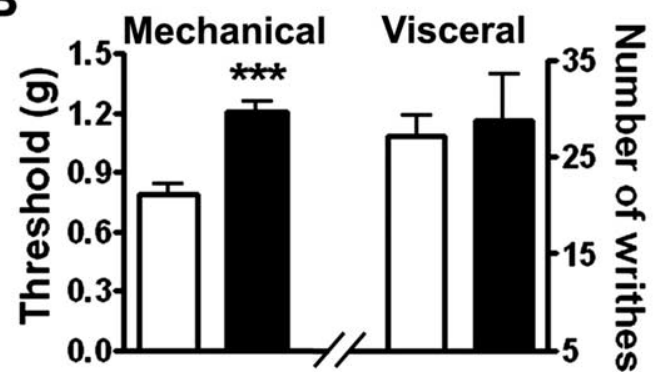

Figure 1. Acute pain and visceral pain tests. $\boldsymbol{A}$, Responses to noxious thermal stimulation, as measured by the tail-flick latency (left) and the paw withdraw latency (Hargreaves, right) induced by radiant heat did not differ between wild-type and $L m \times 1 b^{f / f / p}$ mice. $B$, Left, Pawwithdrawal threshold after exposure to mechanical (von Frey filaments) stimulus was increased in $L m \times 1 b^{f / f / p}$ mice compared with wild-type mice $\left({ }^{* * *} p<0.001\right.$, 2-tailed $t$ test). Right, Visceral pain in response to intraperitoneal injection of $0.6 \%$ acetic acid solution; values represent the number of abdominal stretches (writhes) in 20 min after injection. In all tests, $n=9-15$ per genotype. WT, Wild type.

tected with the Odyssey Infrared Fluorescence Imaging System (LI-COR).

\section{High-performance liquid chromatography}

Two-month-old male $L m x 1 b^{f / f / p}$ mice were divided into four groups for formalin or saline injection and carrageenan or saline injection, respectively. The brain and spinal cord tissues were used for HPLC $(n=5)$. 5-HT and 5-hydroxyindoleacetic acid (5-HIAA) were measured using high-performance liquid chromatography as described previously (Zhao et al., 2006). The resulting values were expressed as picograms of amine per milligram of wet tissue weight.

\section{Statistical analysis}

Statistical comparisons were performed with Student's $t$ test or one- or two-way ANOVA using the statistical software package, Statistica 7 (StatSoft, Tulsa, OK) followed by post hoc tests in behavioral experiments or $t$ test or one-way ANOVA followed by post hoc tests for the morphological and quantitative biochemical comparisons. Except for the mentioned exceptions, all data are expressed as the mean \pm SEM, and error bars represent SEM. In all cases, $p<0.05$ was considered statistically significant.

\section{Results}

Normal basal thermal sensitivity and visceral pain but reduced basal mechanical sensitivity in $L m \times 1 b^{f / f / p}$ mice To determine whether acute nociception was altered in $L m x 1 b^{f / f / p}$ mice, the latency to acute thermal and tactile stimuli was measured using the tail-flick test, the paw-withdrawal (Hargreaves) test, and the von Frey test. Strikingly, the response latencies to radiant heating of the paw and tail were similar in $L m x 1 b^{f / f / p}$ mice and wild-type mice (Fig. $1 A$ ). However, $L m x 1 b^{f / f / p}$ mice showed a significantly higher mechanical withdrawal threshold than wildtype controls as assessed using von Frey filaments (Fig. $1 B$ ). We
A

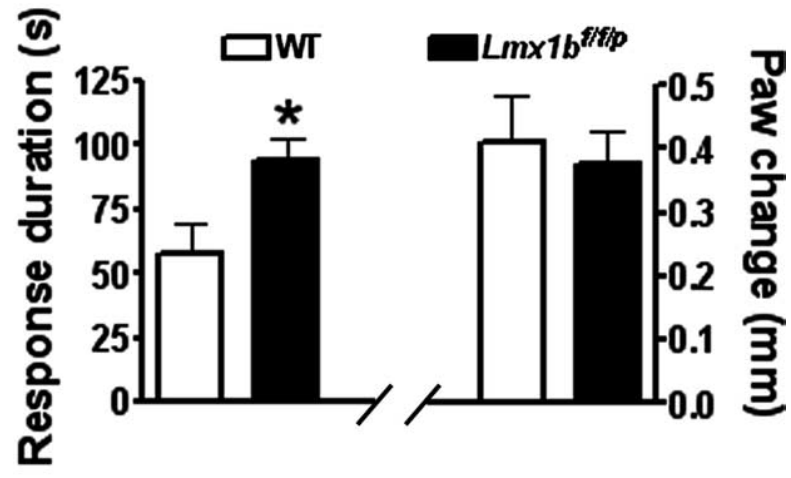

B

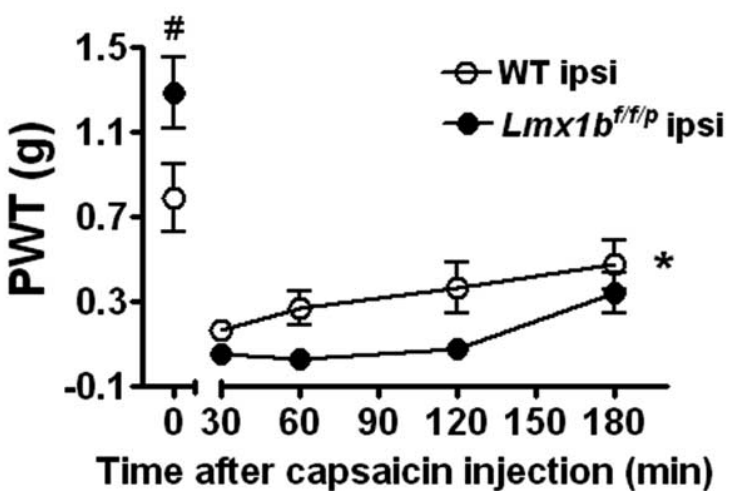

Figure 2. Enhanced pain behavior in $L m \times 1 b^{f / f f p}$ mice after capsaicin injection. $A$, Left, A significant increase in duration of licking and flinching responses within $5 \mathrm{~min}$ after an intraplantar injection of capsaicin in $L m \times 1 b^{f f f / p}$ mice $(n=15)$ compared with wild-type control ( $n=14 ;{ }^{*} p<0.05$, 2-tailed $t$ test). Right, Capsaicin-evoked paw edema measured by the changes of paw diameter $1 \mathrm{~h}$ after capsaicin injection (the value $1 \mathrm{~h}$ after injection minus the value of the same hindpaw before injection) in $L m \times 1 b^{f / f / p}$ mice $(n=9)$ did not differ from wild-type mice $(n=10)$. $\boldsymbol{B}$, Increased mechanical sensitivity measured with von Frey filaments in the ipsilateral hindpaw induced by capsaicin in $L m \times 1 b^{f / f / p}$ mice compared with wild-type mice. ${ }^{\#} p<0.05$, two-tailed $t$ test; ${ }^{*} p<0.05$, repeated two-way ANOVA. Paw-withdrawal mechanical threshold (in grams) of wild-type mice and $L m \times 1 b^{f f / p}$ mice before or from 30 to 180 min after capsaicin injection was measured, respectively $(n=9-12)$. ipsi, Ipsilateral; PWT, paw-withdrawal threshold; WT, wild type.

also examined visceral pain responses in these mice using the acetic acid-induced writhing test and found no significant differences between wild-type and $L m \times 1 b^{f / f / p}$ mice (Fig. $1 B$ ). Altogether, these results demonstrated that $L m \times 1 b^{f / f / p}$ mice have normal basal thermal sensitivity and visceral pain but show reduced basal mechanical sensitivity.

Enhanced inflammatory pain behaviors in $L m x 1 b^{f / f / p}$ mice To determine whether the lack of 5-HT neurons in the CNS alters inflammatory pain responses, we first examined the nocifensive behavioral responses to paw injection of capsaicin in $L m \times 1 b^{f / f / p}$ mice and wild-type mice. Capsaicin evokes a sensation of burning pain and a secondary neurogenic inflammatory pain by activating TRPV1 (transient receptor potential vanilloid-1) (Caterina et al., 1997). Capsaicin was injected into the plantar surface of one of the mouse hindpaws, and the duration of spontaneous nocifensive behavior, defined as licking and flinching of the injected paw, was recorded for $5 \mathrm{~min}$ after the injection. Wild-type mice displayed an immediate short-lasting nocifensive response. However, a significant increase in the duration of licking and flinching behavior was observed in $L m \times 1 b^{f / f / p}$ mice (Fig. $2 A$ ). In 


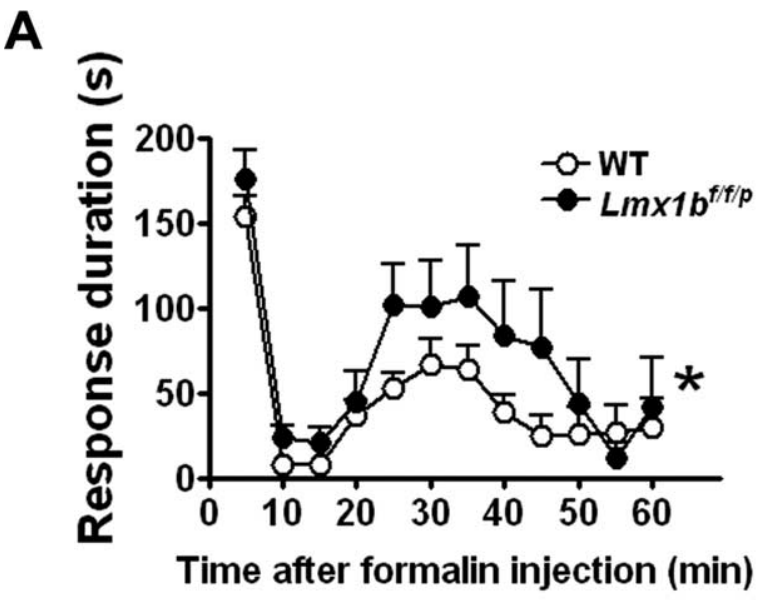

B

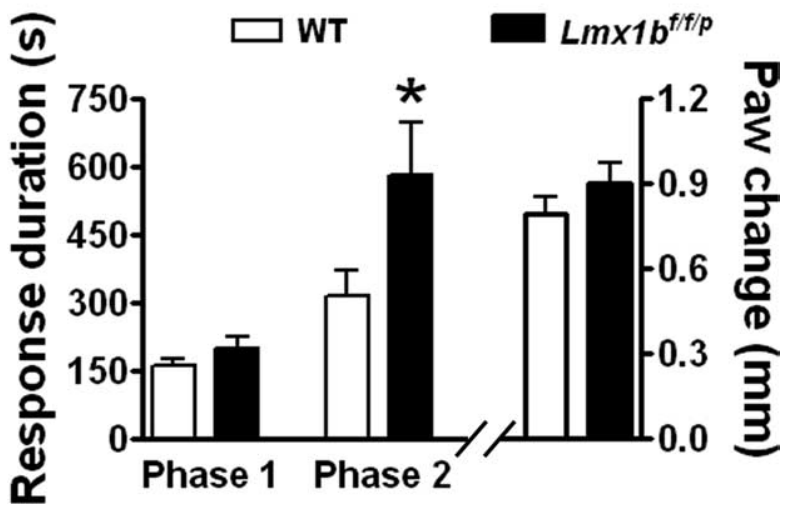

Figure 3. Enhanced pain behavior in $L m \times 1 b^{f / f / p}$ mice after formalin injection. $\boldsymbol{A}$, The first phase ( $0-10 \mathrm{~min}$ ) of pain behavior (as duration of licking and flinching responses) did not differ between wild-type $(n=17)$ and $L m \times 1 b^{f / f / p}$ mice $(n=11)$ after formalin injection, but the second phase (10-50 min) was significantly enhanced in $L m \times 1 b^{f / f / p}$ mice. Data were plotted in 5 min intervals; ${ }^{*} p<0.05$, repeated two-way ANOVA, $L m \times 1 b^{f / f / p}$ versus wild type. $B$, Left, Data from $A$ grouped into two phases; ${ }^{*} p<0.05$; two-tailed $t$ test; $L m \times 1 b^{f / f / p}$ versus wild type. Right, Formalin-evoked paw edema in $L m \times 1 b^{f / f / p}$ mice $(n=11)$ and wild-type mice $(n=17)$ did not differ. WT, Wild type.

addition, mechanical hypersensitivity was observed after capsaicin injection in both wild-type and $L m \times 1 b^{f / f / p}$ mice, but $L m \times 1 b^{f /}$ $f / p$ mice showed significantly greater mechanical hypersensitivity when compared with wild-type mice, indicating an enhanced neurogenic inflammatory pain in the mutants (Fig. $2 B$ ). This enhanced response was not associated with any significant difference in capsaicin-evoked edema, used as an indicator of inflammatory response, between wild-type and $L m \times 1 b^{f / f / p}$ mice (Fig. 2A).

We next examined the responses of $L m \times 1 b^{f / f / p}$ mice in the formalin test, a commonly used test of inflammatory pain in rodents. Subcutaneous injection of formalin normally evokes a biphasic licking and flinching response. The first phase, lasting $\sim 10 \mathrm{~min}$, is believed to result from the activation of nociceptors, whereas the second phase lasts up to $60 \mathrm{~min}$ and may involve central sensitization of dorsal horn neurons (Hunskaar and Hole, 1987). After injection of 5\% formalin into the hindpaw, flinching and licking behaviors of wild-type and $L m \times 1 b^{f / f / p}$ mice were not significantly different during the first phase (Fig. 3). In contrast, licking and flinching responses in the second phase were significantly enhanced in the mutants compared with wild-type littermate controls (Fig. 3A,B). Despite the observed increase in

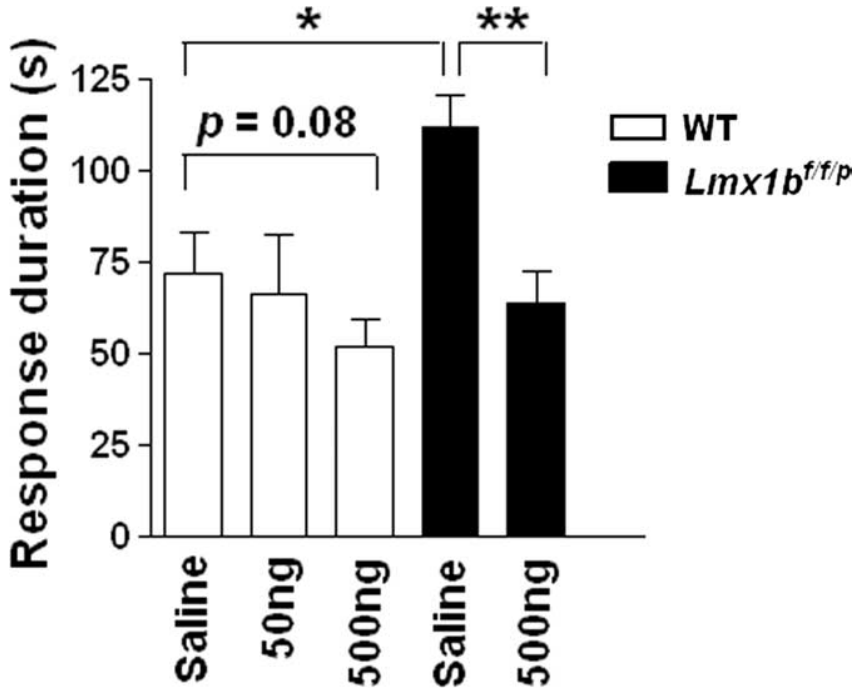

Figure 4. Inhibitory effects of intrathecal injection of different dosages of 5-HT on wild-type and $L m \times 1 b^{f f f / p}$ mice in a capsaicin model of pain. Intrathecal injection of 50 and $500 \mathrm{ng}$ of 5 -HT on wild-type mice reduced the capsaicin-evoked licking and flinching behavior but not significantly compared with the vehicle control. $\operatorname{In} L m \times 1 b^{\mathrm{f} / t / p}$ mice, the capsaicin-induced pain behavior was significantly attenuated by intrathecal injection of $5-\mathrm{HT}(500 \mathrm{ng}) 10 \mathrm{~min}$ before capsaicin injection. ${ }^{*} p<0.05, L m \times 1 b^{f / f / p}$ versus wild type; ${ }^{* *} p<0.01,5$-HT versus vehicle, one-way ANOVA followed by Tukey's posthoc test. $\operatorname{Lm} \times 1 b^{\mathrm{f} / f / p} 500 \mathrm{ng}$ group, $n=13$; all other groups, $n=$ 7 animals. WT, Wild type.

second-phase pain behavior in $L m x 1 b^{f / f / p}$ mice, formalin-evoked paw swelling in these mice did not significantly differ from that in wild-type mice (Fig. $3 B$ ), which also suggested that the central 5 -HT system was not required for the concurrent inflammation.

Enhanced inflammatory pain in $L m x 1 b^{f / f / p}$ mice is attenuated by intrathecal injection of 5-HT

We next wanted to determine whether the enhanced pain responses in $L m \times 1 b^{f / f / p}$ mice can be attenuated by 5 -HT. We performed a rescue experiment by intrathecal injection of 5-HT in $L m \times 1 b^{f / f / p}$ mice. Examination of effects of different dosages of intrathecal 5-HT on wild-type mice before capsaicin injection showed that 5-HT obviously inhibited capsaicin-evoked spontaneous behavior, but the effective doses exceeded $2 \mu \mathrm{g}$ (data not shown). A dose of $500 \mathrm{ng}$ of 5-HT was not capable of reducing capsaicin-induced behavior in wild-type mice (Fig. 4) and was thus chosen for use in mutants. $L m \times 1 b^{f / f / p}$ mice injected with 5-HT followed by capsaicin treatment produced a significant reduction of the licking and flinching behaviors in the absence of obvious tremor activity compared with the mutants injected with vehicle (Fig. 4). This result suggested that the enhanced pain responses in the mutants may be contributed by the lack of 5-HT.

\section{Normal expression of 5-HT receptors in the dorsal horn of} $\operatorname{Lm} x \mathbf{1 b}^{f / f / p}$ mice

Previous studies suggested that 5-HT depletion may alter the expression of 5-HT receptors (Lauder et al., 2000). To determine whether there were changes in 5-HT receptor expression in the mutants, we examined the expression of several 5-HT receptors that have been implicated in the spinal modulation mediated by 5-HT (Bardin et al., 2000; Millan, 2002). Examination of 5- $\mathrm{HT}_{1 \mathrm{~A}}$, $5-\mathrm{HT}_{1 \mathrm{~B}}, 5-\mathrm{HT}_{2 \mathrm{~A}}$, and $5-\mathrm{HT}_{2 \mathrm{C}}$ expression by in situ hybridization in wild-type mice showed that these markers are widely expressed in the dorsal horn of lumbar spinal cord, especially in deep dorsal horn neurons (Fig. 5A-H). No apparent difference in the expres- 


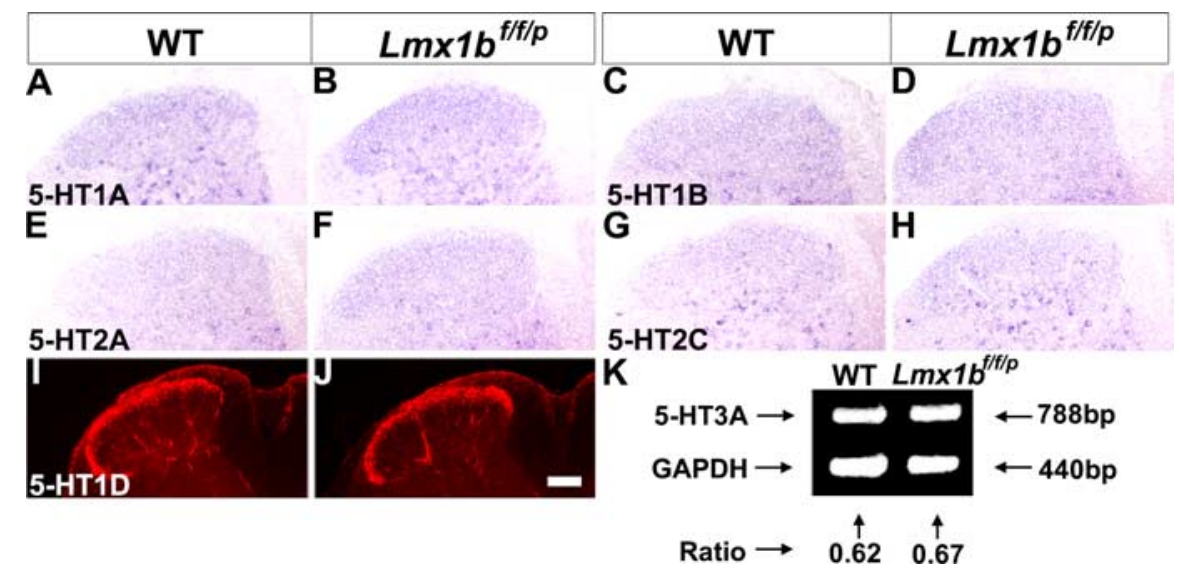

Figure 5. 5-HT receptor expression in the lumbar spinal cord of $L m \times 1 b^{f / f / p}$ mice and wild-type mice. No major difference of 5-HT receptor expression, including $5-\mathrm{HT}_{1 \mathrm{~A}}(\boldsymbol{A}, \boldsymbol{B}), 5-\mathrm{HT}_{1 \mathrm{~B}}(\boldsymbol{C}, \boldsymbol{D}), 5-\mathrm{HT}_{2 \mathrm{~A}}(\boldsymbol{E}, \boldsymbol{F}), 5-\mathrm{HT}_{2 \mathrm{C}}(\boldsymbol{G}, \boldsymbol{H}), 5-\mathrm{HT}_{1 \mathrm{D}}(\boldsymbol{I}, \boldsymbol{J})$, and $5-\mathrm{HT}_{3 \mathrm{~A}}(\boldsymbol{K})$, was found between wild-type (left) and $L m \times 1 b^{f f f / p}$ (right) mice. $\boldsymbol{A}-\boldsymbol{H}$, In situ hybridization data. I, J, Immunocytochemical staining. $\boldsymbol{K}$, Semiquantitative RT-PCR result in the lumbar spinal cord. Scale bar, $100 \mu \mathrm{m}$. WT, Wild type.

A

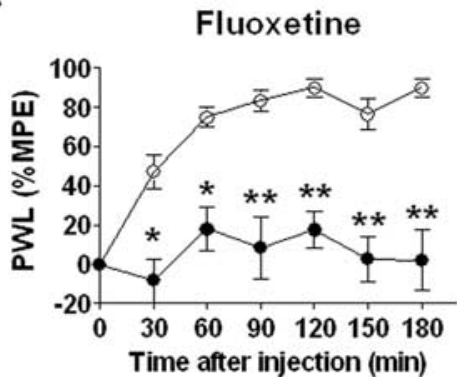

C

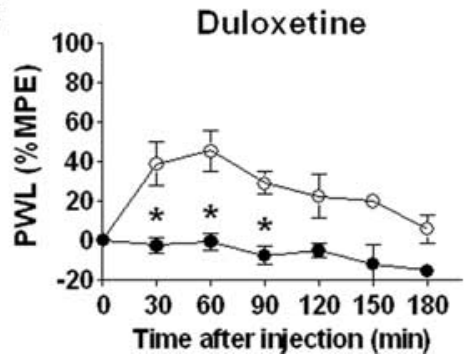

B

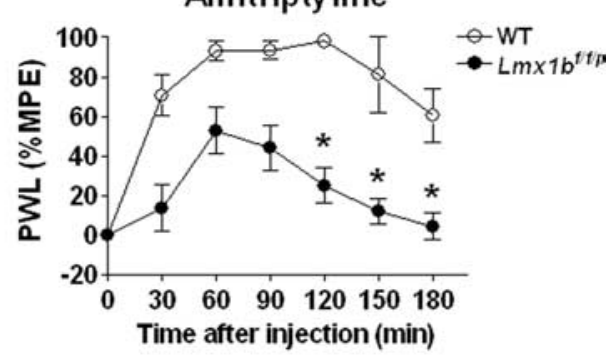

D

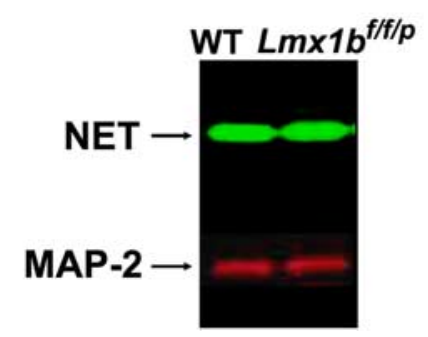

Figure 6. Lack of analgesic efficacy of antidepressants in $L m \times 1 b^{f / f / p}$ mice in the acute thermal pain model. $A$, Fluoxetine injection (20 mg/kg, i.p.) had no effect in $L m \times 1 b^{f / f / p}$ mice $(n=9)$ in the thermal threshold using a Hargreaves-type device, in comparison with that it elicited a strong analgesic effect in wild-type mice $(n=8)$ that lasted $>3 \mathrm{~h}$ after injection. $\boldsymbol{B}$, Amitriptyline injection ( $20 \mathrm{mg} / \mathrm{kg}$, i.p.) elicited a strong analgesic effect in wild-type mice $(n=8)$ that lasted for $2 \mathrm{~h}$ after injection. The analgesic effect of amitriptyline is strongly attenuated in $L m \times 1 b^{f / f / p}$ mice $(n=9)$. C, Duloxetine injection ( $30 \mathrm{mg} / \mathrm{kg}$, i.p.) elicited an analgesic effect in wild-type mice $(n=8)$ that peaked at 60 min after the injection. However, thermal threshold in $L m \times 1 b^{f / f / p}$ mice $(n=7)$ is not affected by duloxetine injection. For each animal, the percentage maximum possible effect (\%MPE) was calculated using the following formula: [(postdrug latency - predrug latency)/(cutoff time - predrug latency)] $\times 100$. ${ }^{*} p<$ $0.05 ;{ }^{* *} p<0.01$, two-way ANOVA followed by Fisher's post hoc test. PWL, Paw-withdrawal latency. D, There were no obvious differences in the levels of NET protein tested by Western blot analyses in the spinal cord of $L m \times 1 b^{f / f / p}$ compared with wild-type mice $(n=4)$. WT, Wild type.

sion of $5-\mathrm{HT}_{1 \mathrm{~A}}, 5-\mathrm{HT}_{1 \mathrm{~B}}, 5-\mathrm{HT}_{2 \mathrm{~A}}$, or $5-\mathrm{HT}_{2 \mathrm{C}}$ receptors was found in $L m \times 1 b^{f f / p}$ mice, compared with wild-type mice (Fig. $5 A-H$ ). In wild-type mice, $5-\mathrm{HT}_{1 \mathrm{D}}$ was expressed in both primary afferents and the dorsal horn as detected by immunocytochemical staining (Fig. 5I), consistent with previous reports (Potrebic et al., 2003). Lmx $1 b^{f f / p}$ mice showed comparable expression of $5-\mathrm{HT}_{1 \mathrm{D}}$ in the superficial dorsal horn (Fig. $5 J$ ). Because of the difficulties of detecting $5-\mathrm{HT}_{3 \mathrm{~A}}$ by in situ hybridization, semiquantitative RT-PCR was performed to measure the level of $5-\mathrm{HT}_{3 \mathrm{~A}}$ in both mutant and wild-type mice. Semiquantitative
RT-PCR results showed no significant difference in $5-\mathrm{HT}_{3 \mathrm{~A}}$ expression in the spinal cord of $L m \times 1 b^{\text {fffp }}$ mice compared with wild-type mice (Fig. $5 K$ ). Together, these results indicate that lack of central 5-HT system does not result in obvious altered 5-HT receptor expression in the dorsal spinal cord.

The analgesic effect of antidepressants is strongly compromised in

$L m x 1 b^{f / f / p}$ mice

Increased 5-HT and/or NE levels are believed to be involved in the antinociceptive action of antidepressant drugs. However, the specific contribution of 5-HT to the analgesic effect of these drugs has not been clear. The complete lack of the central 5-HT system in $L m \times 1 b^{f / f / p}$ mice makes these animals an excellent model to approach this question. We evaluated whether the lack of 5-HT in $L m \times 1 b^{f f / p}$ mice would affect the analgesic properties of different classes of antidepressant drugs when compared with wild-type littermates. Systemic injection of vehicle did not alter thermal sensitivity in either wildtype or $L m \times 1 b^{f f f / p}$ mice (data not shown). Systemic injection of fluoxetine, an SSRI, elicited a strong analgesic effect in wildtype mice that lasted over $3 \mathrm{~h}$ after the injection (Fig. 6A). In contrast, fluoxetine had no effect on thermal thresholds in $L m \times 1 b^{f f / p}$ mice (Fig. 6A). The role of 5 -HT in the analgesic effect of antidepressants that affect both 5-HT and NE reuptake was then evaluated using the TCA amitriptyline and the SNRI duloxetine. Although amitriptyline injection elicited a strong analgesic effect in wild-type mice that lasted for $2 \mathrm{~h}$ after injection, only a mild analgesic effect was observed in $L m \times 1 b^{f f f / p}$ mice (Fig. 6 B). However, when duloxetine was administered, the analgesic effect observed in wild-type mice was completely abolished in $L m x 1 b^{f f / p}$ mice (Fig. 6C). To test whether the absence of the central 5-HT neurons led to an alteration in NET that might account for the lack of analgesic efficacy of duloxetine, we performed Western blots on spinal homogenates from $L m \times 1 b^{f / f / p}$ mice or wildtype mice. We found no difference in NET expression between the two genotypes (Fig. 6D). Together, these results suggest that endogenous central $5-\mathrm{HT}$ system is necessary for the analgesic effect of antidepressant drugs, including those affecting both NE and 5-HT levels in the acute thermal pain model.

There is evidence suggesting that SNRI antidepressants provide increased efficacy in pain relief relative to SSRIs, particularly in models of persistent pain (Iyengar et al., 2004; Mico et al., 2006). To test whether there was a differential involvement of $5-\mathrm{HT}$ in the analgesic effects of SSRIs and SNRIs in persistent 
pain models, we tested the analgesic efficacy of the SSRI fluoxetine and the SNRI duloxetine in $L m \times 1 b^{f / f / p}$ mice and wildtype littermates after formalin injection. In wild-type mice, both fluoxetine and duloxetine significantly reduced the formalin second-phase behaviors, whereas neither significantly impacted the first phase (Fig. $7 A, B)$. In contrast, in $L m \times 1 b^{f / f / p}$ mice, duloxetine had a similar analgesic effect on the second phase, whereas analgesic action of fluoxetine was completely absent (Fig. 7C,D).

Intraplantar injection of carrageenan has been used as a model of more persistent inflammatory pain to evaluate the analgesic efficacy of antidepressants (Jones et al., 2005). We further examined the analgesic effects of antidepressants after carrageenan injection. Mechanical thresholds of the treated paws were assessed before or after carrageenan injection in $L m \times 1 b^{f / f / p}$ mice and wild-type littermates. At $3 \mathrm{~h}$ after carrageenan injection, mice of both genotypes showed significantly increased sensitivity to mechanical stimulation in the ipsilateral hindpaw compared with the vehicle injection (Fig. 8), suggesting an induction of mechanical hypersensitivity by carrageenan. Administration of duloxetine at $30 \mathrm{mg} / \mathrm{kg}$ significantly reversed the mechanical hypersensitivity in both $L m \times 1 b^{f / f / p}$ mice and wild-type littermates compared with vehicle-injected controls (Fig. 8A). In contrast, administration of fluoxetine at $20 \mathrm{mg} / \mathrm{kg}$ only significantly reversed the mechanical hypersensitivity in wild-type mice, but failed to change the mechanical hypersensitivity in $L m \times 1 b^{f / f / p}$ mice compared with vehicle-injected control mice (Fig. $8 \mathrm{~B}$ ).

\section{5-HT and 5-HIAA are not altered in the CNS of $\operatorname{Lm} x 1 b^{f / f / p}$ mice in inflammatory pain}

Serotonin does not cross the blood-brain barrier (BBB) under normal circumstances, but the permeability of the BBB has been shown to increase after acute stress (Sharma et al., 1995; Esposito et al., 2001), possibly because of the activation of mast cells and the release of corticotrophin-releasing hormone (Esposito et al., 2002). To determine whether formalin- or carrageenan-evoked pain behaviors of the mutant mice may be impacted by peripheral 5 -HT because of increased BBB permeability in response to stress in inflammatory states, the concentrations of 5-HT and its metabolite 5-HIAA in the CNS of $L m x 1 b^{f / f / p}$ mice were measured after intraplantar injections of formalin, carrageenan, or vehicle. There were no significant differences in 5-HT and 5-HIAA concentrations in the brain (Fig. 9) or spinal cord (data not shown) when formalin- or carrageenan-treated mice were compared with vehicle-treated controls. This result suggests that it is unlikely that peripheral 5-HT crossing the BBB in response to stress/ pain had a significant impact on altered pain behaviors of Lmx $1 b^{f / f / p}$ mice.

\section{Discussion}

We have previously described the generation of $L m x 1 b^{f / f / p}$ mice lacking transcription factor $L m x 1 b$ only in the central 5-HT neurons (Zhao et al., 2006). In these animals, the central 5-HT system was genetically ablated, yet the mice survived to adulthood and exhibited normal locomotor activity (Zhao et al., 2006). In the present report, we show that $L m \times 1 b^{f / f / p}$ mice have normal acute thermal thresholds and visceral pain responses but are less sensitive to mechanical stimuli and exhibit enhanced inflammatory pain responses. Our studies also reveal the role of central 5-HT system in mediating analgesic effect of several antidepressant drugs.

Central 5-HT system has a key role in facilitating mechanical sensitivity and in inhibiting inflammatory pain

Our findings that $L m \times 1 b^{f / f / p}$ mice showed normal acute visceral pain, and thermal thresholds in the Hargreaves test and the tailflick test suggest that central 5-HT system has minimal influence on these pain tests. Among all of the pain modalities that we have tested, only mechanical thresholds were increased in $L m \times 1 b^{f / f / p}$ mice relative to the wild-type mice. This suggests a facilitatory role of the central 5-HT system in modulating the mechanical sensitivity. Our results provide new in vivo genetic evidence to demonstrate that the central 5-HT system facilitates the nociceptive transmission in response to mechanical stimulation, which has also been described in various neuropathic pain models ( $\mathrm{Su}-$ zuki et al., 2004a; Rahman et al., 2006). Paradoxically, in both capsaicin and carrageenan models, mechanical hypersensitivity was increased in the mutants compared with that in the control mice. Several possibilities may be offered to explain this paradox. Previous reports indicate that the facilitatory role of the 5-HT system is primarily mediated via spinal 5-HT3 receptor, and depletion of spinal 5-HT reduces the mechanical evoked responses of deep dorsal horn neurons (Suzuki et al., 2002; McCleane et al., 2003; Oatway et al., 2004; Rahman et al., 2006). Thus, one plausible explanation for decreased mechanical sensitivity is that the absence of central 5-HT system in $L m \times 1 b^{f / f / p}$ mice may remove activation of facilitatory 5 -HT receptors that tonically modulate 
A

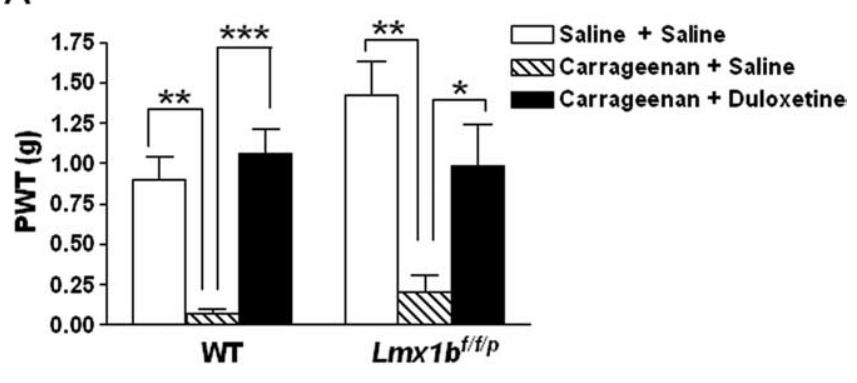

B

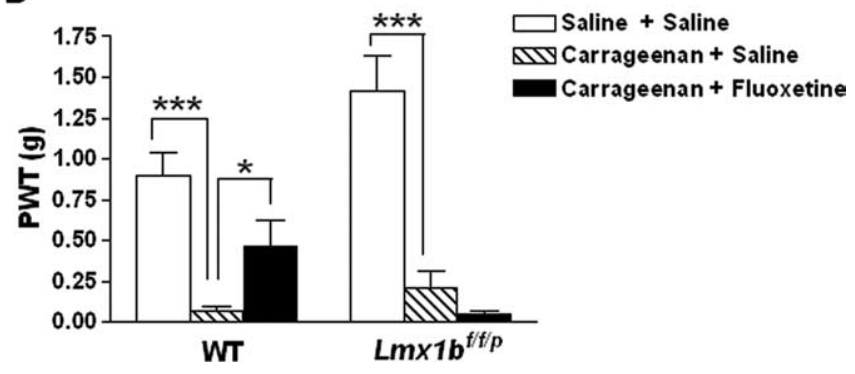

Figure 8. Antidepressants differentially attenuated carrageenan-induced mechanical hypersensitivity in wild-type mice and $L m \times 1 b^{f / f / p}$ mice. $\boldsymbol{A}, \boldsymbol{B}$, Both wild-type mice $(n=8)$ and $L m \times 1 b^{f / f / p}$ mice ( $n=6$ ) developed significantly increased mechanical hypersensitivity as measured by withdrawal threshold in grams to von Frey stimulus, 3 h after intraplantar injection of $2 \%$ carrageenan [intraplantar injection of vehicle (saline) as the control for carrageenan and intraperitoneal injection of vehicle (saline) $1.5 \mathrm{~h}$ after carrageenan as the control for the antidepressants]. $A$, Duloxetine $(30 \mathrm{mg} / \mathrm{kg}$, i.p.) injection $1.5 \mathrm{~h}$ after carrageenan significantly reversed carrageenan-induced mechanical hypersensitivity in both wild-type mice $(n=12)$ and mutants $(n=9)$ when compared with the vehicle contro of the same genotype. $\boldsymbol{B}$, Fluoxetine ( $20 \mathrm{mg} / \mathrm{kg}$, i.p.) significantly reversed mechanical hypersensitivity in wild-type mice $(n=10)$ but failed to reverse carrageenan-induced mechanical hypersensitivity in $L m \times 1 b^{f / f / p}$ mice $(n=7)$ compared with vehicle-injected control of the same genotype. ${ }^{*} p<0.05$; ${ }^{* *} p<0.01$; ${ }^{* * *} p<0.001$, one-way ANOVA followed by Newman-Keuls post hoc test. PWT, Pawwithdrawal threshold; WT, wild type.

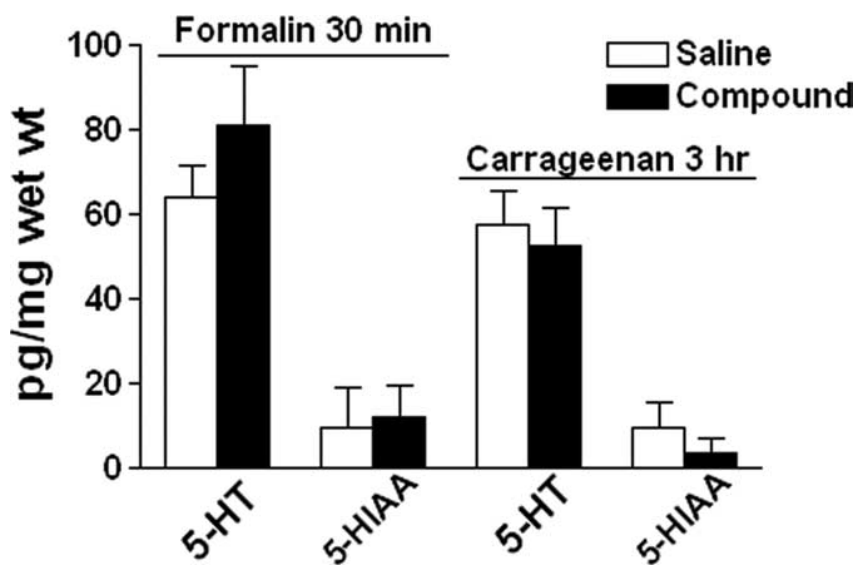

Figure 9. HPLC analysis of 5-HT and 5-HIAA in the brain of $L m \times 1 b^{f / f / p}$ mice after saline vehicle, formalin, and carrageenan injections. There were no significant differences in 5 - $\mathrm{HT}$ or 5 -HIAA concentrations in the brain of $L m \times 1 b^{f / f / p}$ mice 30 min after formalin $(n=5)$ or $3 \mathrm{~h}$ after carrageenan $(n=5)$ injection compared with their respective controls $(n=5)$. One-way ANOVA followed by Newman-Keuls test for comparing the effect of compound with vehicle. wet wt, Wet tissue weight.

mechanical pain thresholds in deep dorsal horn neurons. However, the effect of facilitatory 5-HT receptors in modulation of pain threshold may be counteracted by an upregulated inhibitory mechanism mediated by the central 5 -HT system in inflamma- tory states, resulting in a net effect of the central 5-HT system in repressing the inflammatory pain including mechanical hypersensitivity.

The finding that nocifensive responses and mechanical hypersensitivity after capsaicin injection were increased in $L m \times 1 b^{f / f / p}$ mice relative to wild-type littermates suggests that the central 5-HT neurons normally suppress both the initial response to direct chemical activation of primary afferents and the mechanical hypersensitivity associated with the resulting neurogenic inflammation. Consistent with the alteration in sensitization seen in the capsaicin test, we found that in the formalin model, the time spent engaging in nocifensive behaviors in the second phase was significantly greater for $L m \times 1 b^{f / f / p}$ mice than for their wildtype littermates. However, no differences were observed in the first phase. This lack of the effect on the first phase of the formalin test may seem to contradict the finding of enhanced response to capsaicin in the initial $5 \mathrm{~min}$ after injection. This difference may be the result of differences in the populations of primary afferents activated by the two agents or may be the result of a behavioral ceiling effect in the formalin test during the first few minutes. Finally, the enhanced persistent pain behaviors in $L m x 1 b^{f / f / p}$ mice are most likely caused by the lack of the central 5-HT system rather than peripheral effects, because inflammation induced by capsaicin and formalin and peripheral 5-HT level did not differ between $L m \times 1 b^{f / f / p}$ mice and wild-type littermates, and no increased central 5-HT level caused peripheral 5-HT was found in Lmxlb/f/p mice (Zhao et al., 2006) (Fig. 9).

\section{Differential analgesic effects of various antidepressants in $\operatorname{Lm} x \mathbf{l b}^{f / f / p}$ mice}

Antidepressants have been increasingly used for treating persistent pain, but few genetic animal models were available for studying the mechanism of action of analgesic effect of antidepressants (Mico et al., 2006). In the acute thermal pain test, the analgesic actions of all classes of antidepressants were absent or markedly reduced in the $L m \times 1 b^{f / f / p}$ mice, implicating blockade of 5-HT reuptake as the predominant mechanism of acute analgesic effects of these drugs. In persistent pain states (the formalin and carrageenan models), the analgesic effects of the SNRI duloxetine were not reduced in $L m x 1 b^{f / f / p}$ mice, suggesting that the analgesic effect of duloxetine is independent of the central 5-HT neurons and a potential critical role for $\mathrm{NE}$ in pain and analgesia in these persistent pain models. However, the findings that the analgesic actions of the SSRI fluoxetine were completely absent in these animals confirmed the complete dependence of the SSRI fluoxetine on the central 5-HT neurons for its analgesic effect. Moreover, in wild-type mice, fluoxetine appears to exhibit strong efficacy only in the acute thermal pain model (Fig. 6), whereas its analgesic effects appear to be less efficacious in the persistent pain models (Figs. 7, 8), suggesting that the role of central 5-HT varies in different settings of pain. Together, these findings are consistent with the literature indicating that dual-acting reuptake inhibitors are superior to the SSRIs for treating persistent pain conditions in humans (Briley, 2004; Bomholt et al., 2005; Maizels and McCarberg, 2005).

Antidepressants may elicit their analgesic effect acting as local anesthetics through peripheral mechanisms such as sodium channel and the blockade of 5-HT receptors (Sawynok et al., 2001; Strumper and Durieux, 2004; Groves, 2006). Because peripheral 5-HT is primarily pronociceptive (Sommer, 2004), it is less likely that it may contribute to the analgesic action of antidepressants. Could peripheral 5-HT account for some aspects of altered pain behavior of $L m \times 1 b^{f / f / p}$ mice via either counteracting 
or facilitating the central 5-HT effect? Our previous studies suggested that peripheral 5 -HT is not affected in $L m \times 1 b^{f / f / p}$ mice (Zhao et al., 2006). The present data showed that there was no increased 5-HT concentration in the CNS of the mutants caused by the penetration of peripheral 5-HT in inflammatory states. Thus, our results suggest that peripheral 5-HT is not involved in the analgesic effects of antidepressants. These results highlight the importance of using an animal model in which the central 5 -HT system is genetically ablated to determine the contribution of peripheral versus central 5-HT system to the analgesic effect of antidepressant drugs.

Although the noradrenergic system in $L m \times 1 b^{f / f / p}$ mice appears to be normal, the analgesic effect of the TCA amitriptyline on acute thermal pain behavior was strongly attenuated in $L m x 1 b^{f / f / p}$ mice. Because a total absence of analgesic effect was observed in $L m \times 1 b^{f / f / p}$ mice treated with fluoxetine and duloxetine, the residual analgesic effect observed in $L m \times 1 b^{f / f / p}$ mice treated with amitriptyline is likely caused by mechanisms other than blockade of 5-HT and NE reuptake, such as channel modulation and NMDA receptor antagonism (Lawson, 2002; Wang et al., 2004). Together, our data indicate that although the NE component seems to be critical in the analgesic effect of antidepressants, endogenous 5-HT is also of fundamental importance for the analgesic effect of these drugs, especially in reducing thermal sensitivity.

\section{Does plasticity play a role in pain behaviors of $\operatorname{Lm} x \mathbf{l b}^{f / f / p}$ mice?}

Pain is intimately associated with other behavioral states that are likely to be regulated by 5-HT neurons (Mason, 2001; Gingrich et al., 2003). Although the analgesic effect of antidepressants is believed to be independent of their antidepressant effect (Stahl and Briley, 2004), one may argue that an altered behavioral state caused by ablation of 5-HT neurons could contribute to the altered nociceptive behavior in a variety of ways. Lack of central 5-HT neurons in $L m \times 1 b^{f / f / p}$ mice may interfere with the normal function of these other neurotransmitters, which have been shown to be tightly associated with 5-HT (Bolte Taylor et al., 1998; Zhou et al., 2005). Moreover, adaptive changes or plasticity of the CNS may exist in $L m \times 1 b^{f / f / p}$ mice because of the loss of central 5-HT neurons during development. Finally, central 5-HT neurons produce not only 5-HT but also a number of painrelated neuropeptides and receptors, which may contribute to the plasticity of the brain. Despite these possibilities, it is important to note that our results in large degree support pharmacological studies indicating that the central 5-HT neurons play a major inhibitory role in inflammatory pain, and antidepressants differentially depend on these 5-HT neurons. Thus, the present work has validated the utility of $L m \times 1 b^{f / f / p}$ mice as a unique animal model for studying the function of central 5-HT neurons in painrelated behavior. Future temporal conditional knock-out studies will be necessary to define whether there are adaptive changes resulting from the lack of the central 5-HT neurons in brain development, and if so, to what degree such changes may contribute to altered pain behaviors or the analgesic effect of antidepressants.

\section{References}

Bardin L, Lavarenne J, Eschalier A (2000) Serotonin receptor subtypes involved in the spinal antinociceptive effect of 5-HT in rats. Pain 86:11-18. Basbaum AI, Fields HL (1984) Endogenous pain control systems: brainstem spinal pathways and endorphin circuitry. Annu Rev Neurosci 7:309-338.

Bolte Taylor J, Cunningham MC, Benes FM (1998) Neonatal raphe lesions increase dopamine fibers in prefrontal cortex of adult rats. NeuroReport 9:1811-1815.
Bomholt SF, Mikkelsen JD, Blackburn-Munro G (2005) Antinociceptive effects of the antidepressants amitriptyline, duloxetine, mirtazapine and citalopram in animal models of acute, persistent and neuropathic pain. Neuropharmacology 48:252-263.

Briley M (2004) Clinical experience with dual action antidepressants in different chronic pain syndromes. Hum Psychopharmacol 19 [Suppl 1]:S21-S25.

Cao YQ, Mantyh PW, Carlson EJ, Gillespie AM, Epstein CJ, Basbaum AI (1998) Primary afferent tachykinins are required to experience moderate to intense pain [see comments]. Nature 392:390-394.

Caterina MJ, Schumacher MA, Tominaga M, Rosen TA, Levine JD, Julius D (1997) The capsaicin receptor: a heat-activated ion channel in the pain pathway [see comments]. Nature 389:816-824.

Collins SL, Moore RA, McQuay HJ, Wiffen P (2000) Antidepressants and anticonvulsants for diabetic neuropathy and postherpetic neuralgia: a quantitative systematic review. J Pain Symptom Manage 20:449-458.

Ding YQ, Marklund U, Yuan W, Yin J, Wegman L, Ericson J, Deneris E, Johnson RL, Chen ZF (2003) Lmxlb is essential for the development of serotonergic neurons. Nat Neurosci 6:933-938.

Esposito P, Gheorghe D, Kandere K, Pang X, Connolly R, Jacobson S, Theoharides TC (2001) Acute stress increases permeability of the bloodbrain-barrier through activation of brain mast cells. Brain Res 888:117-127.

Esposito P, Chandler N, Kandere K, Basu S, Jacobson S, Connolly R, Tutor D, Theoharides TC (2002) Corticotropin-releasing hormone and brain mast cells regulate blood-brain-barrier permeability induced by acute stress. J Pharmacol Exp Ther 303:1061-1066.

Fields HL, Basbaum AI (1978) Brainstem control of spinal paintransmission neurons. Annu Rev Physiol 40:217-248.

Fields HL, Heinricher MM, Mason P (1991) Neurotransmitters in nociceptive modulatory circuits. Annu Rev Neurosci 14:219-245.

Fishbain DA, Cutler R, Rosomoff HL, Rosomoff RS (2000) Evidence-based data from animal and human experimental studies on pain relief with antidepressants: a structured review. Pain Med 1:310-316.

Gingrich JA, Ansorge MS, Merker R, Weisstaub N, Zhou M (2003) New lessons from knockout mice: the role of serotonin during development and its possible contribution to the origins of neuropsychiatric disorders. CNS Spectr 8:572-577.

Groves DS (2006) Antidepressants as local anesthetics: is there a place in regional anesthesia? Curr Opin Anaesthesiol 19:516-520.

Hamon MD, Collin E, Chantrel D, Verge D, Bourgoin S (1991) The contribution of monoamines and their receptors to pain control. In: Towards a new pharmacotherapy of pain (Basbaum AI, Besson JM, eds), pp 83-102. Chichester, UK: Wiley.

Hargreaves K, Dubner R, Brown F, Flores C, Joris J (1988) A new and sensitive method for measuring thermal nociception in cutaneous hyperalgesia. Pain 32:77-88.

Hunskaar S, Hole K (1987) The formalin test in mice: dissociation between inflammatory and non-inflammatory pain. Pain 30:103-114.

Iyengar S, Webster AA, Hemrick-Luecke SK, Xu JY, Simmons RM (2004) Efficacy of duloxetine, a potent and balanced serotonin-norepinephrine reuptake inhibitor in persistent pain models in rats. J Pharmacol Exp Ther 311:576-584

Jones CK, Peters SC, Shannon HE (2005) Efficacy of duloxetine, a potent and balanced serotonergic and noradrenergic reuptake inhibitor, in inflammatory and acute pain models in rodents. J Pharmacol Exp Ther 312:726-732.

Lauder JM, Liu J, Grayson DR (2000) In utero exposure to serotonergic drugs alters neonatal expression of 5-HT(1A) receptor transcripts: a quantitative RT-PCR study. Int J Dev Neurosci 18:171-176.

Lawson K (2002) Tricyclic antidepressants and fibromyalgia: what is the mechanism of action? Expert Opin Investig Drugs 11:1437-1445.

Le Bars D (1988) Serotonin and pain. In: Neuronal serotonin (Osborne NN, Hamon M, eds), pp 171-229. Chichester, UK: Wiley.

Lynch ME (2001) Antidepressants as analgesics: a review of randomized controlled trials. J Psychiatry Neurosci 26:30-36.

Maizels M, McCarberg B (2005) Antidepressants and antiepileptic drugs for chronic non-cancer pain. Am Fam Physician 71:483-490.

Mason P (2001) Contributions of the medullary raphe and ventromedial reticular region to pain modulation and other homeostatic functions. Annu Rev Neurosci 24:737-777.

Max MB, Lynch SA, Muir J, Shoaf SE, Smoller B, Dubner R (1992) Effects of 
desipramine, amitriptyline, and fluoxetine on pain in diabetic neuropathy. N Engl J Med 326:1250-1256.

McCleane GJ, Suzuki R, Dickenson AH (2003) Does a single intravenous injection of the 5HT3 receptor antagonist ondansetron have an analgesic effect in neuropathic pain? A double-blinded, placebo-controlled crossover study. Anesth Analg 97:1474-1478.

Mico JA, Ardid D, Berrocoso E, Eschalier A (2006) Antidepressants and pain. Trends Pharmacol Sci 27:348-354.

Millan MJ (2002) Descending control of pain. Prog Neurobiol 66:355-474.

Oatway MA, Chen Y, Weaver LC (2004) The 5-HT3 receptor facilitates atlevel mechanical allodynia following spinal cord injury. Pain 110:259-268.

Potrebic S, Ahn AH, Skinner K, Fields HL, Basbaum AI (2003) Peptidergic nociceptors of both trigeminal and dorsal root ganglia express serotonin 1D receptors: implications for the selective antimigraine action of triptans. J Neurosci 23:10988-10997.

Rahman W, Suzuki R, Webber M, Hunt SP, Dickenson AH (2006) Depletion of endogenous spinal 5-HT attenuates the behavioural hypersensitivity to mechanical and cooling stimuli induced by spinal nerve ligation. Pain 123:264-274.

Sawynok J, Esser MJ, Reid AR (2001) Antidepressants as analgesics: an overview of central and peripheral mechanisms of action. J Psychiatry Neurosci 26:21-29.

Sharma HS, Westman J, Navarro JC, Dey PK, Nyberg F (1995) Probable involvement of serotonin in the increased permeability of the blood-brain barrier by forced swimming. An experimental study using Evans blue and 131I-sodium tracers in the rat. Behav Brain Res 72:189-196.

Sindrup SH, Jensen TS (1999) Efficacy of pharmacological treatments of neuropathic pain: an update and effect related to mechanism of drug action. Pain 83:389-400.

Singh VP, Jain NK, Kulkarni SK (2001) On the antinociceptive effect of fluoxetine, a selective serotonin reuptake inhibitor. Brain Res 915:218-226.
Sommer C (2004) Serotonin in pain and analgesia: actions in the periphery. Mol Neurobiol 30:117-125.

Stahl S, Briley M (2004) Understanding pain in depression. Hum Psychopharmacol 19 [Suppl 1]:S9-S13.

Strumper D, Durieux ME (2004) Antidepressants as long-acting local anesthetics. Reg Anesth Pain Med 29:277-285.

Suzuki R, Morcuende S, Webber M, Hunt SP, Dickenson AH (2002) Superficial NK1-expressing neurons control spinal excitability through activation of descending pathways. Nat Neurosci 5:1319-1326.

Suzuki R, Rahman W, Hunt SP, Dickenson AH (2004a) Descending facilitatory control of mechanically evoked responses is enhanced in deep dorsal horn neurones following peripheral nerve injury. Brain Res 1019:68-76.

Suzuki R, Rygh LJ, Dickenson AH (2004b) Bad news from the brain: descending 5-HT pathways that control spinal pain processing. Trends Pharmacol Sci 25:613-617.

Urban MO, Gebhart GF (1999) Supraspinal contributions to hyperalgesia. Proc Natl Acad Sci USA 96:7687-7692.

Wang GK, Russell C, Wang SY (2004) State-dependent block of voltagegated $\mathrm{Na}+$ channels by amitriptyline via the local anesthetic receptor and its implication for neuropathic pain. Pain 110:166-174.

Wernicke JF, Pritchett YL, D’Souza DN, Waninger A, Tran P, Iyengar S, Raskin J (2006) A randomized controlled trial of duloxetine in diabetic peripheral neuropathic pain. Neurology 67:1411-1420.

Zhao ZQ, Scott M, Chiechio S, Wang JS, Renner KJ, Gereau IV RW, Johnson RL, Deneris ES, Chen ZF (2006) Lmxlb is required for maintenance of central serotonergic neurons and mice lacking central serotonergic system exhibit normal locomotor activity. J Neurosci 26:12781-12788.

Zhou FM, Liang Y, Salas R, Zhang L, De Biasi M, Dani JA (2005) Corelease of dopamine and serotonin from striatal dopamine terminals. Neuron 46:65-74.

Zhuo M, Gebhart GF (1991) Spinal serotonin receptors mediate descending facilitation of a nociceptive reflex from the nuclei reticularis gigantocellularis and gigantocellularis pars alpha in the rat. Brain Res 550:35-48. 